\title{
Cross talk between physical activity and appetite control: does physical activity stimulate appetite?
}

\author{
J. E. Blundell ${ }^{*}$, R. J. Stubbs 2 , D. A. Hughes ${ }^{2}$, S. Whybrow ${ }^{2}$ and N. A. King ${ }^{1}$ \\ ${ }^{1}$ BioPsychology Group, School of Psychology, University of Leeds, Leeds LS2 9JT, UK \\ ${ }^{2}$ Human Nutrition Group, Rowett Research Institute, Aberdeen AB21 9SB, UK
}

\begin{abstract}
Physical activity has the potential to modulate appetite control by improving the sensitivity of the physiological satiety signalling system, by adjusting macronutrient preferences or food choices and by altering the hedonic response to food. There is evidence for all these actions. Concerning the impact of physical activity on energy balance, there exists a belief that physical activity drives up hunger and increases food intake, thereby rendering it futile as a method of weight control. There is, however, no evidence for such an immediate or automatic effect. Short (1-2 d)-term and medium (7-16 d)-term studies demonstrate that men and women can tolerate substantial negative energy balances of $\leq 4 \mathrm{MJ}$ energy cost/d when performing physical activity programmes. Consequently, the immediate effect of taking up exercise is weight loss (although this outcome is sometimes difficult to assess due to changes in body composition or fluid compartmentalization). However, subsequently food intake begins to increase in order to provide compensation for about $30 \%$ of the energy expended in activity. This compensation (up to $16 \mathrm{~d}$ ) is partial and incomplete. Moreover, subjects separate into compensators and non-compensators. The exact nature of these differences in compensation and whether it is actually reflective of non-compliance with protocols is yet to be determined. Some subjects (men and women) performing activity with a cost of $\leq 4 \mathrm{MJ} / \mathrm{d}$ for $14 \mathrm{~d}$, show no change in daily energy intake. Conversely, it can be demonstrated that when active individuals are forced into a sedentary routine food intake does not decrease to a lower level to match the reduced energy expenditure. Consequently, this situation creates a substantial positive energy balance accompanied by weight gain. The next stage is to further characterize the compensators and non-compensators, and to identify the mechanisms (physiological or behavioural) that are responsible for the rate of compensation and its limits.
\end{abstract}

\section{Energy balance: Physical activity: Appetite}

For over 50 years the relationship between energy expenditure (EE) from physical activity and energy intake (EI) has been a central theme running through research on energy balance (EB). Indeed, some researchers have surmised that EB is achieved through the impact of EE on the mechanisms of appetite control (for example, see Edholm et al. 1955). However, the nature of the relationship between EE and EI has remained elusive. One initial idea of a $2 \mathrm{~d}$ lag between EE and EI (Edholm et al. 1955) was abandoned when research showed that 'there appears to be no detectable pattern of relationship between expenditures and intakes during the same or following days (Edholm et al. 1970). It was also noted that 'there was no confirmation of the 2 day lag between expenditure and intake' (Edholm, 1977). In recent years several reviews have been published on this general theme (Blundell \& King, 1996, 1998; King et al. 1997b; King, 1998). A major problem in this area has been the immense difficulty with which precise and accurate estimates of day-to-day changes in EI and EE can be made.

It is likely that the adoption of a more active lifestyle will have a number of consequences for appetite control. The outcome can be evaluated experimentally by examination of the sensitivity of EI compensation to exercise-induced changes in EB. However, it is important to recognize the difference between acute and long-term interventions on the one hand, and established (habitual) patterns of behaviour on the other. It is also important to note that the behaviour of subjects in many short- and medium-term interventions may reflect habitual behaviour in everyday life, rather than behavioural responses to the actual intervention (Stubbs 
et al. 1998). Often in acute studies all aspects of the energy budget cannot be measured. However, changes in the components of appetite control can be measured, as a consequence of specific interventions. For example, it has recently been demonstrated that compensation for a high-carbohydrate preload is more accurate in habitual exercisers than non-exercisers (Long et al. 2002). Following an acute bout of physical activity subjects are better able to discriminate between the energy values of so-called energyrich and non-energy-rich beverages (King et al. 1999). Consequently, physical activity may improve the sensitivity of satiety signalling systems to consumed food.

Physical activity could also alter macronutrient preferences and food choices; this response might be expected as a drive to seek particular foods to replenish short-term energy stores, and might be reflected in the food quotient $\left(\mathrm{CO}_{2}\right.$ produced: $\mathrm{O}_{2}$ consumed during oxidation of the food) of the diet selected following episodes of physical activity (for example, see Tremblay et al. 1985; King \& Blundell, 1995). Alternatively, this effect could be detected via preferences for particular tastes associated with certain nutrients, e.g. sweetness, that would normally be associated with carbohydrate energy needed to replace the glycogen used in exercise (for example, see Westerterp-Plantenga et al. 1997).

Physical activity may influence appetite by modulating the hedonic response to foods, i.e. individuals would report greater pleasure from food consumption following a period of exercise. This phenomenon has been demonstrated for female dieters who showed increased ratings of pleasantness for a range of foods after exercise (Lluch et al. 1998).

These examples illustrate that there is evidence that physical activity can improve the sensitivity of the appetite control system, adjust macronutrient preferences or food choices and enhance the pleasure derived from food consumption. However, there is still a large gap in the literature between these acute studies and the longer-term interventions or cross-sectional studies that encompass the time window in which most compensation is likely to occur. There are a number of key issues pertaining to the relationship between EE and EI in the medium term (1-4 weeks) that are addressed in the present paper: (1) the effect of increasing exercise-induced $\mathrm{EE}$ on appetite, energy and nutrient intake in the short, medium and long term; (2) the nature, rate and extent of compensation of EB for exerciseinduced changes in EE; (3) the effect of a decrease in activity on energy and nutrient intake; (4) the implications for weight control of responses in different modalities to increases or decreases in activity.

\section{What is the effect of increasing exercise-induced energy expenditure on appetite, energy and nutrient intake in the short, medium and long term?}

\section{Acute effects of exercise on hunger and energy intake}

To examine the issue of acute compensatory changes in hunger and food intake short-term intervention studies have been conducted that deliberately impose acute bouts of exercise with subsequent measurement of food (energy) intake. Contrary to a widespread belief, there is no short-term compensatory increase in hunger and food intake (Reger et al. 1984; Thompson et al. 1988; Kissileff et al. 1990; King et al. 1994, 1996, 1997a; King \& Blundell, 1995; Imbeault et al. 1997; Westerterp-Plantenga et al. 1997; Lluch et al. 1998). Overall, the body of evidence points to a loose coupling between exercise-induced EE and EI (for reviews, see King et al. 1997a; Blundell \& King, 1998; King, 1998) over these time frames.

A criticism of the short-term interventions is that the exercised-induced increment in EE was not large enough to stimulate appetite and the EI was not tracked for sufficiently long enough following the increased physical activity. However, even with a high dose of exercise (gross exerciseinduced increase in EE 4.6 MJ) and tracking EI for $2 \mathrm{~d}$, there is no automatic compensatory rise in hunger and EI (King et al. 1997a). Thus, the evidence that suggests an acute exercise-induced negative EB is not compensated by an increase in EI is fairly robust.

These results are counterintuitive, particularly when short-term reductions in EI (another method of inducing a short-term negative EB) give rise to increases in hunger and food intake (Lawton et al. 1993; Green et al. 1994; Delargy et al. 1995; Hubert et al. 1998). These two methods of inducing a short-term negative EB have been compared in a single study using a within-subjects design. EI was manipulated at breakfast (breakfast $v$. no breakfast) followed by an activity manipulation (exercise $v$. no exercise). The results demonstrated that failing to eat (when a meal is usually taken) induced marked increases in hunger and EI, whereas exercise (imposed when it normally would not have occurred) had no effect on hunger and EI (Hubert et al. 1998). Hunger was greater following no breakfast compared with breakfast, whereas there was no difference in hunger between the exercise and no-exercise treatments. Similarly, volunteers ate more at the test lunch following the nobreakfast treatment, independent of the exercise treatment.

Despite a lack of an effect on EI per se in these studies, there was a marked effect of intense exercise on subjective hunger. This phenomenon has been termed exercise-induced anorexia but only occurs during, and for a short time following, vigorous (>60\% maximun $\mathrm{O}_{2}$ consumption) exercise (Thompson et al. 1988; Kissileff et al. 1990; King et al. 1994, 1996; King \& Blundell, 1995; WesterterpPlantenga et al. 1997). The suppression of hunger is short lived and is not seen with moderate- or low-intensity exercise. It is known that during exercise there is a considerable redistribution of blood flow. In particular, there is a redirection of flow away from the splanchnic circulation, towards the muscles. This response may well be implicated in the phenomenon of exercise-induced anorexia.

Thus, in the very short term very intense bouts of exercise can induce a suppression of hunger. However, the main feature of acute bouts of exercise on appetite and EI is the marked lack of an effect. Clearly, this form of response cannot continue indefinitely.

\section{Medium-term effects of exercise on hunger and energy intake}

While there is a good deal of literature relating to the effects of various modes, intensities and levels of physical activity 
on patterns of feeding or on estimates of EI, fewer studies have sought to quantify the relationship between the three components of the EB equation (EI, EE and EB) in subjects with ad libitum access to food undergoing various exercise interventions. Some studies are not experimental interventions examining the relationship between EI and EE, rather they exploit specific situations such as training in athletes (for example, see Van Etten et al. 1997; Westerterp, 1998; Van Baak, 1999). Under these conditions EI may not be entirely ad libitum. Other studies are almost entirely observational in nature and therefore do not constitute an exercise intervention. These studies are important and need to be considered in relation to intervention studies. However, life is never simple. Of the studies that have examined the effect of exercise interventions on patterns of feeding and/or EI, methodologies have varied considerably (for review, see Blundell \& King, 1998). Studies have employed varying exercise loads ranging from $0.4 \mathrm{MJ} / \mathrm{d}$ (Durrant \& Royston, 1982) to $>10 \mathrm{MJ} / \mathrm{d}$ (Brouns et al. 1989). Type and intensity of exercise vary considerably between protocols, as does the extent to which the components of EB are measured or estimated. The net effect of these methodological differences is that the literature has become peppered with diverse results using various methodologies, different subjects and experimental protocols. While these studies are individually valuable, it is not surprising to find from the literature that exercise can increase, decrease or have no effect on patterns of EI (King et al. 1997a). Few studies have examined systematically the relationship between changes in EE and feeding behaviour in normally-sedentary non-obese subjects who do not have a preconceived goal associated with weight reduction or a training programme. The review of King et al. (1997a) of the effects of exercise regimens on appetite and/or EI shows that in short- to medium-term intervention studies (often no longer than 2-5d) $19 \%$ report an increase in EI after exercise, $65 \%$ show no change and $16 \%$ show a decrease. Longer-term studies that measure body composition suggest some fat mass is lost but lean body mass tends to be preserved in response to exercise regimens, depending on the absolute level of EB. There are fewer data in the literature on how changes in EI respond to alterations in EE over periods of 1-2 weeks.

To overcome some of these problems it is important that a number of studies should be interrelated, using similar methodologies that can easily be compared, in order to understand the relationship between changing EE through physical activity and patterns of food intake with reference to the general population. A few laboratories have conducted a structured series of studies that have specifically assessed the effect of exercise-induced changes in $\mathrm{EE}$ on patterns of energy and nutrient intake and subjective appetite. For example, Woo and colleagues (Woo et al. 1982a,b; Woo \& Pi-Sunyer, 1985) have conducted a series of three studies under metabolic balance conditions, ranging from 19 to $57 \mathrm{~d}$, in lean and overweight women. Other researchers have also conducted a number of acute interventions (for example, see Imbeault et al. 1997) and assessments of the longer-term effects of training programmes on feeding behaviour and EB (for example, see Tremblay et al. 1985). A series of studies, using methodologies that allow comparison between these and previous studies was therefore designed.

In an initial study the effect of graded increases in exercised-induced $\mathrm{EE}$ on appetite, EI, total daily EE and body weight were examined, over $7 \mathrm{~d}$ per treatment, in six lean women living in their normal environment (Stubbs et al. 2002b). Subjects were each studied three times during a $9 \mathrm{~d}$ protocol, corresponding to no exercise (Nex; $0 \mathrm{MJ} / \mathrm{d}$ ), medium exercise level (Mex; approximately $1.3 \mathrm{MJ} / \mathrm{d}$ ) and high exercise level (Hex; approximately $2.6 \mathrm{MJ} / \mathrm{d}$ ). On days 1-7 the subjects self recorded ad libitum intake of their usual diet using a food diary and self-weighed intake. EE amounted to $9 \cdot 2,11 \cdot 0$ and $12 \cdot 1 \mathrm{MJ} / \mathrm{d}$ on the Nex, Mex and Hex treatments respectively. The corresponding values for EI were $8.9,9.2$ and $10.0 \mathrm{MJ} / \mathrm{d}$. There was no treatment effect on hunger, appetite or body weight, but weight loss was different from zero on the Mex and Hex treatments.

This same study was repeated in six men. EE amounted to $11.7,12.9$ and $16.8 \mathrm{MJ} / \mathrm{d}$ on the Nex, Mex and Hex treatments respectively. The corresponding values for EI were $11.6,11.8$ and $11.8 \mathrm{MJ} / \mathrm{d}$. There were no treatment effects on hunger, appetite or body weight, but there was evidence of weight loss on the Hex treatment.

In both studies a graded increase in EE due to exercise markedly elevated total daily EE, which in turn precipitated a negative $\mathrm{EB}$ that was roughly proportional to the energy cost of exercise. In both groups daily EE declined during the course of the study on the exercise regimens. This response suggests an approximate 'compensation' of approximately $0.3-0.6 \mathrm{MJ} / \mathrm{d}$ on the Hex regimen and $0.3-0.4 \mathrm{MJ} / \mathrm{d}$ on the Mex regimen in both men and women. Thus, the greatest tendency toward restoration of EB in these studies occurred due to a decrease in total daily EE (excluding the energy cost of exercise), as each study progressed (Stubbs et al. 2002a, $b$; Fig. 1).

Combining the data sets from both studies allowed a comparison of the responses of men and women to the same graded-exercise intervention. There was a gender effect for EI and EE. On average, across all treatments, men expended $13.8 \mathrm{MJ} / \mathrm{d}$ while women expended $10.7 \mathrm{MJ} / \mathrm{d}$. There was a tendency for women to compensate through EI and the tendency for the men's EE to exceed the prescribed energy cost of exercise, especially on the Hex treatment. For the women exercise improved some aspects of mood, which was not the case for the men.

The findings from these two studies suggested that over a period of $7 \mathrm{~d}$ EI does not really track a marked elevation of EE. A further study extended these protocols, using the same exercise prescription, to a $14 \mathrm{~d}$ exercise intervention in six men and six women. EB was assessed with greater precision (Stubbs et al. 2003c). EE was estimated using doubly-labelled water (instead of heart-rate monitoring used in the previous two studies). EI was quantified by providing subjects with a diet of constant measurable composition (the same as that used in previous feeding behaviour studies; for example, see Stubbs et al. 2000). This particular study was designed to continually monitor these variables, together with subjective appetite, in the same men and women exposed to Nex-, Mex- or Hex-induced EE, under 'pseudo free-living' conditions (Stubbs et al. 2003c). 

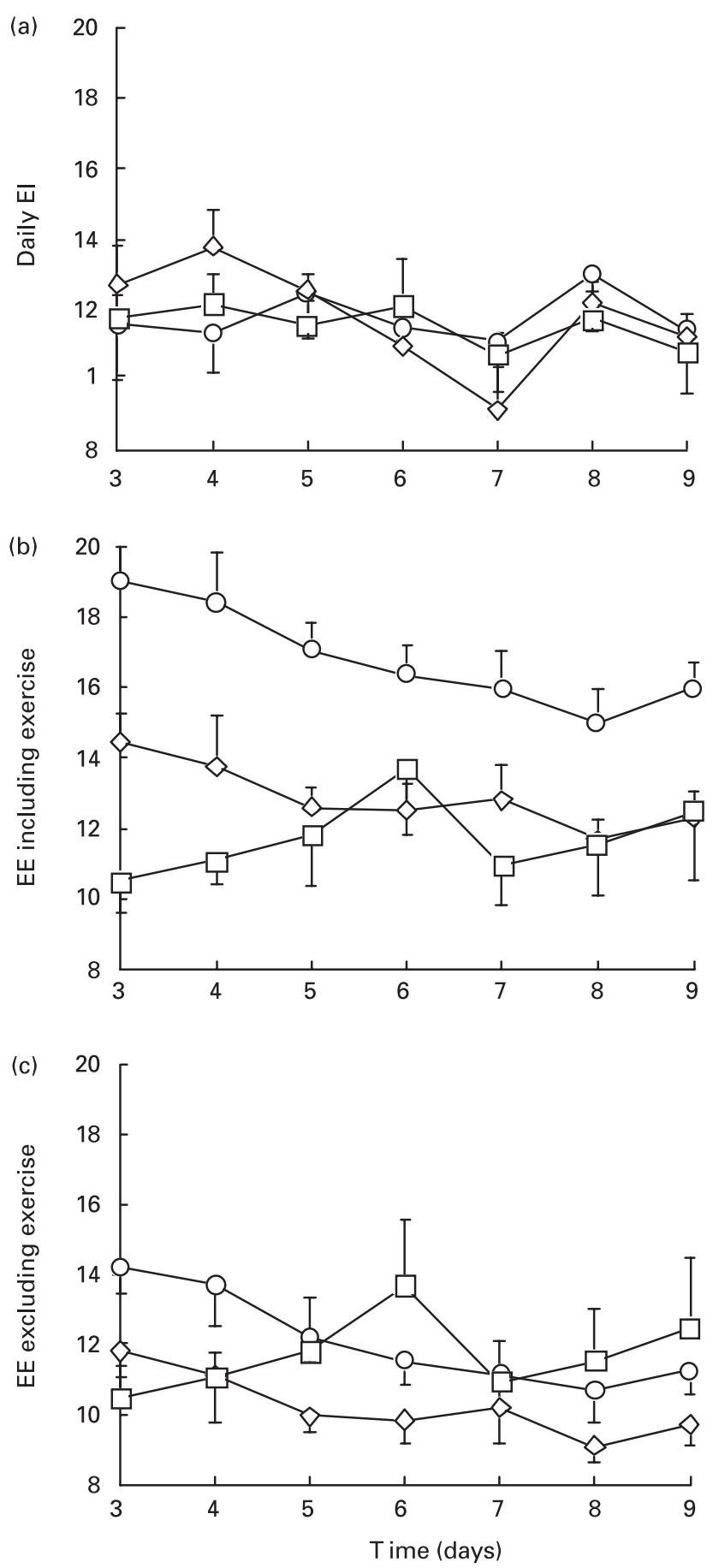

Fig. 1. (a) Mean daily energy intake (EI), (b) energy expenditure (EE) including exercise and (c) EE excluding exercise for the six men on the three exercise treatments; $\square$, (no-exercise (Nex; $0 \mathrm{MJ} / \mathrm{d}$ ); $\diamond$, medium exercise level (Mex; approximately $1.3 \mathrm{MJ} / \mathrm{d}$ ); $\bigcirc$, high exercise level (Hex; approximately $2.6 \mathrm{MJ} / \mathrm{d})$ ). For details of procedures, see p. 653 . Values are means with their standard errors represented by vertical bars. EE including and excluding exercise declined from days 3-9 on the Hex and Mex treatments. The data clearly show that subjects appeared to eat in relation to their normal (Nex) activity routine.

EE amounted to $9 \cdot 2,11.6$ and $13.7 \mathrm{MJ} / \mathrm{d}$ for the women and $12.2,14.0$ and $16.7 \mathrm{MJ} / \mathrm{d}$ for the men on the Nex, Mex and Hex treatments respectively. The corresponding EI were

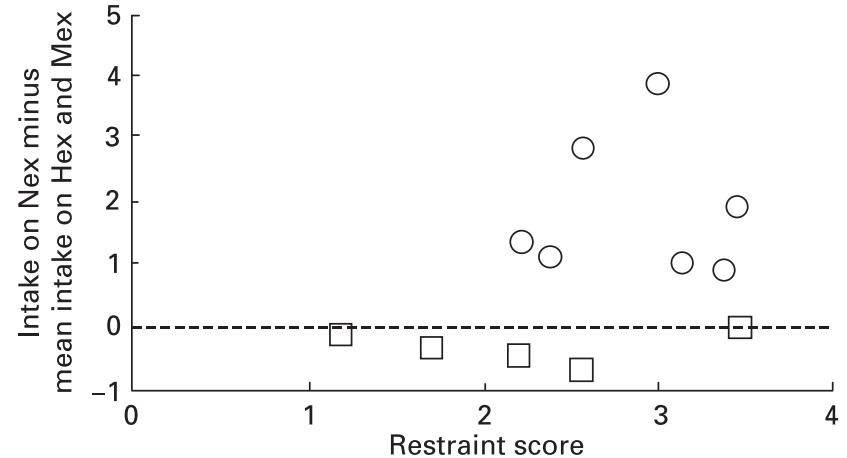

Fig. 2. Scatter plot of the average (within-subject) difference between intake on no exercise $(\mathrm{Nex} ; 0 \mathrm{MJ} / \mathrm{d})$ and the mean of the two exercise treatments (medium exercise level (Mex; approximately $1 \cdot 3 \mathrm{MJ} / \mathrm{d}$ ) and high exercise level (Hex; approximately $2 \cdot 6 \mathrm{MJ} / \mathrm{d})$ ) $v$. restraint score (the conscious or deliberate control over eating) for the three women and two men termed 'non-compensators' ( $\square$ ) and the three women and four men termed 'compensators' (O). For details of procedures, see p. 653.

$8 \cdot 3,8.6$ and $9.9 \mathrm{MJ} / \mathrm{d}$ for the women and $10 \cdot 6,11.6$ and $12.0 \mathrm{MJ} / \mathrm{d}$ for the men. On average subjects compensated for approximately $30 \%$ of the exercise-induced energy deficit. Irrespective of gender, there was considerable variation in the extent of compensation. On examining the data individually it became apparent that some subjects did not appear to change EI at all between the Nex and the Hex treatments (i.e. the lowest energy deficit $v$. the highest energy deficit). These data are shown in Fig. 2.

'Compensators' (three women and four men) are here defined as subjects who did increase their EI on going from the Nex treatment to the Hex treatment. 'Noncompensators' (three women and two men) are defined as those who showed no increase in EI on going from the Nex treatment to the Hex treatment. There was a difference in the way the two groups responded, with the 'compensators' having an average EI of $9 \cdot 1,10.4$ and $11.6 \mathrm{MJ} / \mathrm{d}$ on the Nex, Mex and Hex treatments respectively, increasing across treatments. By definition, the 'non-compensators' showed no significant increase in EI across treatments, with averages of $10 \cdot 0,9.3$ and $10.0 \mathrm{MJ} / \mathrm{d}$ on the Nex, Mex and Hex treatments respectively. The resulting change in body weight for the exercise intervention period were, -0.68 , $0.96,-1.09 \mathrm{~kg}$ for the 'compensators' and $-0.47,-0.60$, $-0.57 \mathrm{~kg}$ for 'non-compensators' on the Nex, Mex and Hex treatments respectively. The dietary restraint scores (the conscious or deliberate control over eating) for the 'compensators' and 'non-compensators' were 2.9 and 2.2 respectively. Exactly what it is about those subjects who did compensate and those who did not is an important issue yet to be resolved. The body-weight data actually suggest that the 'non-compensators' were simply not as compliant with their diets. Thus, while the scatter plot in Fig. 2 suggests two distinct groups, the number of subjects in each group is far too small to make clear statements about different compensatory responses to exercise. At present our view is that the difference between the two groups may relate more to compliance with instructions than to the extent of 
behavioural or physiological compensatory responses to exercise-induced energy deficits. However, such an effect would have considerable practical importance. The resolution of this issue is important and will require studies with far more subjects than were included in this protocol.

\section{Why is compensation for exercise-induced energy deficits so slow?}

This study and our previous studies appear to capture the first stages of a change in EI in order to match a markedly elevated EE. Presumably it takes a number of weeks for EI and EE to achieve a new balance. Indeed, what is remarkable about these data is not the extent to which the subjects compensated, but the extent to which they did not compensate and so tolerated a marked negative EB over periods amounting to $14 \mathrm{~d}$.

Certain factors associated with the exercise regimen itself may limit the extent to which subjects compensate food intake in the medium term. In the studies described earlier there may be a specific increase in fluid intake on going from the Nex treatment to the Hex treatments. Clearly, when exercising at this level water turnover increases. Part of the lack of marked compensation of EI for a considerable exercise-induced negative EB may be due to the priority given to increasing fluid intake to maintain fluid balance. Water balance is more tightly defended than is EB (Stricker \& Verbalis, 1999; Stricker, 2000). As soon as the exercise period terminated (i.e. in the post-exercise period) the increased fluid intake subsided. Thus, it may be that part of the slow response of EI to increases in EE is due to the need to give priority to defending fluid balance over EB. It has become apparent also that the response of EE to exerciseinduced increases in $\mathrm{EE}$ may also be a quantitatively important component of EB compensation.

\section{Long-term effects of exercise on hunger and energy intake}

There is growing evidence that it takes considerable time for EI to adjust to elevations of EE. One direct intervention also supports this view. It is important to recognize that because fatness constrains fitness, many interventions in the obese will fail because substantially elevating daily EE through exercise is too arduous. Thus, in order to examine the longterm effects of an increase in EE on EI this problem has to be overcome. Lim \& Lee (1994) studied the impact of 5 months of basic military training on body weight, body fat and lean body mass in 197 Singapore males, classified as normalweight (BMI $24-<30 \mathrm{~kg} / \mathrm{m}^{2}$ ), obese (BMI $30-<35 \mathrm{~kg} / \mathrm{m}^{2}$ ) and very obese $\left(\mathrm{BMI}>35 \mathrm{~kg} / \mathrm{m}^{2}\right)$. Two key features of this study are that training was incremental, allowing subjects to gradually become fitter, and food intake was ad libitum. Over the 5 months of training fat-free mass did not change but subjects lost substantial amounts of weight and body fat. Subjects who were initially fatter lost more weight and more fat. This finding suggests that responses of EI to exerciseinduced changes in EE may depend on how fat you are (Fig. 3). In other words it is likely that fat mass is acting as an energy buffer and EI rises markedly when lean body mass is threatened by the exercise-induced energy deficit.

The importance of a change in lean body mass for appetite control has been discussed by Stubbs \& Elia (2001). It appears that substantial fat loss is possible before EI begins to track a sustained elevation of EE.

It is also important to ask how much exercise is required to maintain weight loss, subsequent to weight reduction. Two studies have examined how much EE is required to sustain weight loss, i.e. to prevent weight regain (Schoeller et al. 1997; Saris, 2002). The estimates from the two studies are not dissimilar and suggest it takes approximately $40-47 \mathrm{~kJ}$ additional EE through physical activity/kg per $\mathrm{d}$ to prevent weight regain. This finding suggests that there are some powerful regulatory processes opposing the maintenance of weight loss (Schoeller et al. 1997; Saris, 2002).

\section{What is the nature, rate and extent of compensation of energy balance for exercise-induced changes in energy expenditure?}

A major paradox in the field of appetite and EB is that a range of manipulations of diet and exercise can exert a profound effect on EB in the short to medium term. It is not difficult to grossly perturb EB over periods of 1-2 weeks using diet and exercise (for a discussion, see Blundell \&
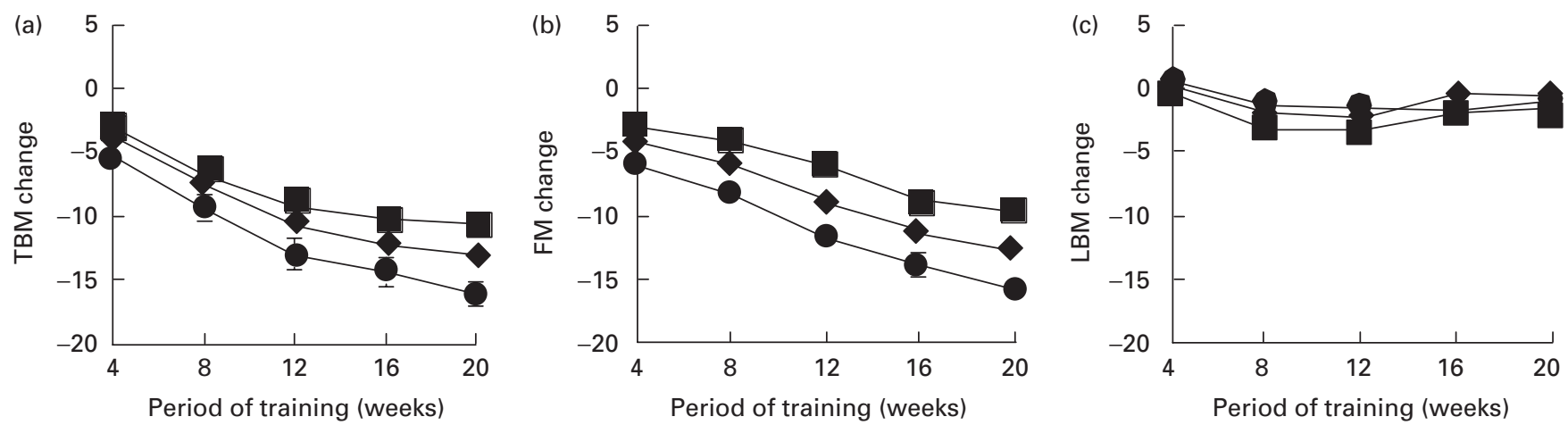

Fig. 3. Changes in (a) total body weight (TBM), (b) fat mass (FM) and (c) lean body mass (LBM) in response to 5 months incremental military training amongst 197 male recruits to the Singapore armed forces, classified as normal-weight (BMl 24-<30 kg/m²; $\mathbf{\square})$, obese (BMI $\left.30-<35 \mathrm{~kg} / \mathrm{m}^{2} ; \bullet\right)$ and very obese $\left(\mathrm{BMl}<35 \mathrm{~kg} / \mathrm{m}^{2} ; \bullet\right)$. Values are means with their standard errors represented by vertical bars. (From Lim \& Lee, 1994.) 
Stubbs, 1998). However, when these interventions have been used in the long term to manipulate EB (almost always in a negative direction), they inevitable have relatively little impact (for example, see Verboeket-van de Venne et al. 1996; Kelly et al. 1998). This finding raised two further issues. First, is the nature of compensation of EB symmetrical? Second, what is the rate and extent of compensation for altered $\mathrm{EB}$ ?

The first question has two aspects. The nature of compensation of EB is clearly not symmetrical. There is overwhelmingly a priori evidence that man, as a species, tolerates energy surfeits far more that energy deficits (Blundell \& Stubbs, 1998; Stubbs et al. 2002a,b); hence the current epidemic of overweight and obesity (Seidell, 2002). It is, however, unclear which components of EB compensate and to what extent. In the first two studies looking at compensation of EI for changes in EE, it was initially surprising to find greater compensation due to changes in non-exercise EE, than due to any change in EI. Concerning the rate and extent of compensation of EB, there is also $a$ priori evidence that while individuals compensate in the long term they do not do so in the short term.

Diets were covertly manipulated (Stubbs et al. 2001, $2002 a, b)$ in order to examine the combined effects of increases in dietary fat content (and energy density) and exercise on EB. Evidence for the day-to-day rate of compensation of either EI or EE over $7 \mathrm{~d}$ was then examined for these initial perturbations of EB.

The effects of Nex and Hex (approximately $4 \mathrm{MJ} / \mathrm{d}$ ) and two dietary manipulations, a high-fat diet and a low-fat diet, on compensatory changes in EI and EE over $7 \mathrm{~d}$ periods were assessed. Eight lean men were each studied four times, in a $2 \times 2$ design. Mean daily EE was 17.6 and $11.5 \mathrm{MJ} / \mathrm{d}$ on the pooled Hex and Nex treatments respectively. EI was higher on high-fat diets $(13.4 \mathrm{MJ} / \mathrm{d}$, pooled) compared with the low-fat diets $(9.0 \mathrm{MJ} / \mathrm{d})$. Regression analysis showed that these energy imbalances induced compensatory changes in EB over time of approximately 0.3-0.4 MJ/d.

On regression of EI, EE and EB $v$. study day some interesting patterns emerged, which shed light on the rate and nature of compensation for induced energy imbalances. In relation to EI, subjects decreased EI by $-0.22 \mathrm{MJ} / \mathrm{d}$ as a response to the higher EI with the high-fat diet. No such compensatory response was seen with the low-fat diet. While weak, this finding suggests that subjects would have compensated by $1.5 \mathrm{MJ} /$ week. It can be estimated that it would take approximately 4 weeks for the difference in EB to be reduced to zero if this compensatory trend continued at a linear rate. A similar trend occurred on the Nex treatment, compared with the Hex treatment, but due to the variability in the data this effect was not significant. There was no compensation of EI over time on the Hex treatment. This lack of response may be due to the tendency for subjects to defend fluid balance over EB. In addition, it may well be that a large part of the reduction in $\mathrm{EE}$ was due to a fatigue effect. It may be they were too tired over the course of the study to compensate EI further.

Compensatory trends were more marked for changes in $\mathrm{EE}$ than for changes in EI in response to the exercise conditions. The daily decrease in $\mathrm{EE}$ of $0.32 \mathrm{MJ} / \mathrm{d}$ (or $2 \cdot 2 \mathrm{MJ} /$ week) on the Hex treatment was largely due to a progressive decrease in non-exercise $\mathrm{EE}$ as the week progressed. A large component of this decrease appears to have been due to an increase in time spent resting during non-exercise activities. This finding suggests that a period of 2-4 weeks would be required at this rate in order for the difference in EB between the two treatments $(5 \cdot 3 \mathrm{MJ} / \mathrm{d})$ to decrease to zero. It is notable that subjects also decreased daily EE over time on the low-fat diet as compared with the high-fat diet. It is on the low-fat diet that EI were far below those required to meet $\mathrm{EB}$. This finding suggests that the low level of EI limited the capacity for these subjects to expend energy through exercise. It is known that at far lower levels of EI (e.g. semi starvation and starvation) physical activity decreases, and this response has a sparing effect on total EB (Keys, 1950; Gibney, 2001; Johnstone, 2001). In the present study subjects would have had to eat approximately $7 \mathrm{~kg} / \mathrm{d}$ to achieve EB on the low-fat Hex treatment. This requirement would clearly create a conflict between the mandatory exercise regimen and the need to eat to EB. Thus, the conditions that produced a marked negative EB at the outset of the study (Hex, low-fat) led to a trend towards

(a)

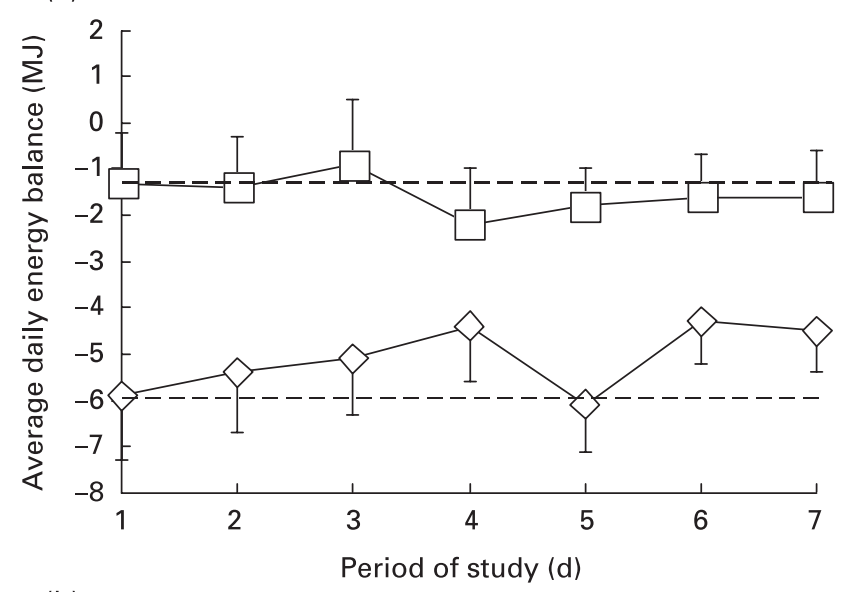

(b)

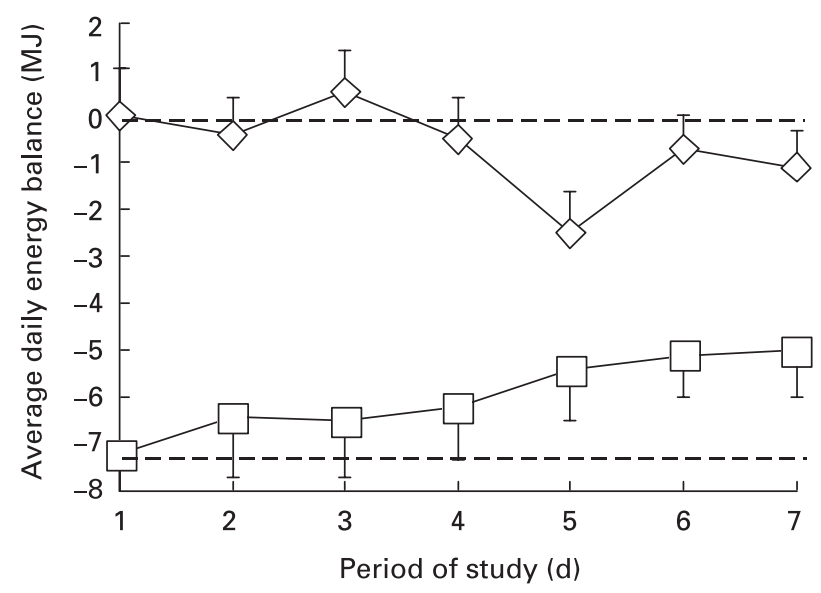

Fig. 4. Average daily energy balance for each day of the study examining responses to perturbations of energy balance (EB) induced by (a) diet $(\diamond$, low-fat diet; $\square$, high-fat diet) and (b) exercise $(\diamond$, no exercise; $\square$, high level of exercise (approximately $4 \mathrm{MJ} / \mathrm{d}$ )). For details of procedures, see p. 656 . Values are means with their standard errors represented by vertical bars. 
restoring EB as the study progressed (Fig. 4). The effect induced by no exercise and that of a high-fat diet (which would both tend to elevate EB) was far less apparent but in the opposite direction. Together these data suggest that the subjects' EB adjusts more readily in response to energy deficits than to energy surfeits. The compensatory changes are due to changes in both EE (at this level of activity) and EI.

The estimates of rate of adjustment, whilst imperfect, are of particular importance because they suggest a time window of compensation of 2-4 weeks if the rate of readjustment continues to increase at a linear rate. The majority of studies examining the effects of diet on exercise make inferences about compensation over periods of $\leq 2$ weeks or $>4$ weeks (including cross-sectional studies). It is important to note that the rate of compensatory change in EB will most likely be a function of the initial rate of change of EB (Gibney, 2001; Johnstone, 2001). Thus, our estimate of the rate of EB restoration primarily pertains to certain particular experimental conditions and may well not apply to other subjects or other conditions. These observations may help explain the apparent paradox whereby the majority of short-term studies show virtually no compensation for changes in dietary energy density or exerciseinduced changes in EE, and why the majority of long-term and cross-sectional studies show far greater compensation of EI in relation to habitual EE (for a discussion, see Blundell \& Stubbs, 1998). There are far fewer long-term dietary manipulations, but they too show greater compensation over the long term (Verboeket-van de Venne et al. 1996; Kelly et al. 1998).

It is important to fully appreciate the nature of compensation of EB in human subjects. It is known that during altered EB there are a number of small but important changes in the components of EE. These changes include: (1) tissuedependent changes in sleeping and BMR (Elia et al. 1992); (2) changes in the energy cost of weight change alter as weight is gained or lost (Forbes et al. 1982; Elia et al. 1992); (3) exercise efficiency will alter as weight changes (Shephard \& Lavallee, 1978); (4) as weight is gained or lost the energy cost of weight-bearing activities will change (Maldonado et al. 2002); (5) during substantive overfeeding, diet composition (fat $v$. carbohydrate) will influence the energy cost of nutrient storage by approximately $15 \%$ (Horton et al. 1995). An important question is what the aggregate effect of these changes is on overall EB. The responses (1-5) are all 'obligatory' responses. The extent of inter-individual variability in these responses will define the scope within which various mechanisms of EB compensation can operate. The extent of inter-individual variability in these responses is not clear (Stubbs et al. 2003a).

In addition to these obligatory changes, altered EB can stimulate active behavioural responses, which are presumably the consequence of cross talk between EI and EE. Altered EB will lead to changes in the mode, duration and intensity of physical activities, including postural and positional activities. In addition to changes in EE, the size, frequency and composition of ingestive events can also change. Some of these changes will be mediated by changes in appetite mentioned earlier. The relative importance of 'obligatory' $v$. facultative behavioural responses, as components of EB control, need to be defined. Further insights into the nature of compensation of EB can be obtained by examining the responses to decreases in activity.

\section{What is the effect of a decrease in activity on energy and nutrient intake?}

Average daily EE appears to be less than was typical of the population several decades ago (Health Education Authority and Sports Council, 1992). It is generally accepted that Western populations are more sedentary than they used to be. Black et al. (1996) have estimated, from tracer studies, that the established limits of total daily EE range from 1.2 to $4.5 \times$ BMR over periods of $\geq 2$ weeks (Black et al. 1996). Total EE for intense sustained activity tends to be a little lower for most individuals at approximately $2 \cdot 3-2 \cdot 9 \times \mathrm{BMR}$. The general (rather sedentary) population appears to be less variable, with the average total daily $\mathrm{EE}$ in the range 1.6-1.8 $\times$ BMR (Black et al. 1996). These results raise the important question of whether there is much scope to markedly affect EB by reducing physical activity within the normal range, given that modern lifestyles are apparently so sedentary.

The effects of inactivity on appetite and/or EI have been assessed in disease (Schoeller, 1998), in $6^{\circ}$ head-down-tilt bed rest (as a model of zero gravity; Ritz et al. 1999) and as a consequence of life in space itself (Lane et al. 1997). In addition, the effect of imposing a sedentary routine on healthy subjects has been examined in the short-term. For instance, Murgatroyd et al. (1999) conducted 2 d treatments in which subjects underwent active $v$. sedentary routines and consumed high-fat $v$. low-fat diets, in a $2 \times 2$ design. It was noted that on a given diet subjects consumed the same level of energy regardless of the level of EE. Similarly, Shephard et al. (2001) have noticed that when lean and obese subjects were placed in the calorimeter for $1 \mathrm{~d}$ the decrease in activity associated with the sedentary environment of the calorimeter generated a positive EB. However, these assessments are very short and King et al. (1997b) have noted that studies of this duration, in general, have produced no change in feeding behaviour, under a variety of conditions. In another of our studies total daily EE was therefore clamped at approximately 1.4 and $1.8 \times$ BMR and the impact of this treatment on motivation to eat and EI was examined for seven consecutive days, while continually resident in a whole-body indirect calorimeter (Stubbs et al. 2003b). Six lean men were each studied twice in a $9 \mathrm{~d}$ protocol, corresponding to a sedentary $(1.4 \times$ BMR $)$ and moderately active $(1.8 \times$ BMR $)$ regimen. EE amounted to 9.7 and $12.8 \mathrm{MJ} / \mathrm{d}$ on the sedentary and active treatments respectively. The corresponding values for EI were 13.5 and $14.4 \mathrm{MJ} / \mathrm{d}$ respectively. There was no treatment effect on hunger, appetite or body weight. By day 7 cumulative EB was $11 \cdot 1$ and $26.3 \mathrm{MJ}$ respectively and most of the excess energy was stored as fat (Fig. 5). Regression analysis showed that EI did not change over time in response to a positive EB. These results are in contrast to the studies conducted in men and women in which subjects did respond to energy deficits over time. In response to imposed sedentariness subjects did not decrease $\mathrm{EE}$ or increase EI at all. This finding suggests that human subjects are more tolerant of positive than negative EB. 


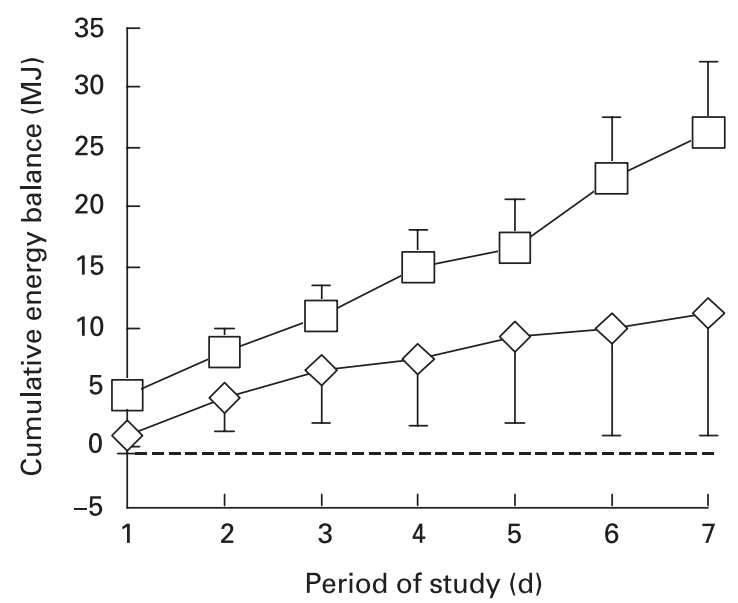

Fig. 5. Mean cumulative energy balance (MJ) for six men on the moderately-active $(1.8 \times \mathrm{BMR} ; \diamond)$ and sedentary $(1.4 \times \mathrm{BMR} ; \square)$ treatments, between days 1 and 7 of treatment, while continually resident in a whole-body indirect calorimeter and feeding ad libitum. Values are means with their standard errors represented by vertical bars.

From the perspective of public health, there are a number of cross-sectional studies that strongly suggest sedentariness is highly correlated with increased adiposity. For example, Martinez-Gonzalez et al. (1999) estimated the association between leisure-time sedentary and non-sedentary selfreported activities on BMI and the prevalence of obesity in 15239 subjects across the fifteen member states of the EU. They found that '...obesity and a higher body weight are strongly associated with a sedentary lifestyle and a lack of physical activity in the adult population of the European Union'. They even go as far as to suggest that a reduction in EE in leisure time may be the main determinant of the current epidemic of obesity. The data in the present study (Stubbs et al. 2003b) suggest that a decrease in physical activity (to $1.4 \times$ BMR) has considerable scope to decrease $\mathrm{EE}$ and that there is no apparent compensation of EI for the decrease. This finding is not new and has been reported elsewhere in short-term studies (Murgatroyd et al. 1999; Shephard et al. 2001). However, the lack of any time trend in EI over the $7 \mathrm{~d}$ of this study suggests that in the short to medium term there is no tendency for EI to begin to decrease in line with a consistent reduction in EE due to activity. Thus, an increase in sedentariness has considerable scope to decrease EE and hence generate a positive EB.

\section{What are the implications of different responses to increases or decreases in activity, for weight control?}

The implications of the earlier findings for weight control are illustrated by a further small study conducted in middle-aged women. The National Health and Nutrition Education Survey data sets suggest that adult human subjects tend to gain weight at a rate of about $0 \cdot 2-2 \mathrm{~kg} / \mathrm{year}$ (Kant et al. 1995). As such a large proportion of Western populations are overweight rather than clinically obese, there is sound logic in public health initiatives aimed at inducing mild to moderate energy deficits, which in the long term may off-set the tendency to overeat and gain weight at the rates suggested earlier. The prevention of a $0 \cdot 2-2 \mathrm{~kg}$ weight gain/year should promote wide-scale weight stability. Nevertheless, how easy is it to induce even small energy deficits over a period of weeks in subjects who are vulnerable to weight gain? It would appear that one of the most vulnerable groups in this respect are women, especially as they become older, typically between 30 and 50 years of age. Theoretically at least, this group should also be more motivated to achieve such energy deficits, since it is young to middle-aged women who are the mainstay of the weight-control industry. There has been considerable emphasis in recent years on the need to engage in frequent mild to moderate exercise as a major means of reducing risk of cardiovascular disease and weight gain. For instance, the World Health Organization's (1990) recommendations for physical activity states 'aerobic activity sustained for periods of at least 20 minutes, three to five times per week at a level between $50 \%$ and $85 \%$ of the maximum oxygen capacity is appropriate' for tangible benefits to health, well-being and fitness. It is critical to assess how easy it is for vulnerable groups to engage in such exercise regimens, and even if they do, what the impact is on their body weight. The effect of a 4 -week exercise intervention (prescribed at approximately $1.5 \mathrm{MJ} / \mathrm{d}, 5 \mathrm{~d} /$ week) on feeding behaviour, body weight and composition was therefore investigated in nine normal-weight and eight overweight women (Hughes et al. 2003). The results of the study were remarkable, in that subjecting lean and overweight women to exercise levels recommended by the World Health Organization (1990) had little discernible effect on total daily EE. Furthermore, there was no significant effect on selfreported EI, or on EB as assessed by changes in body weight and composition, over the 4-week intervention period. The reasons for the lack of any notable effect on EI, daily EE or EB over 4 weeks requires some discussion, since there is an ongoing debate as to the usefulness of exercise as a means of controlling body weight (Garrow \& Summerbell, 1995; Miller et al. 1997). Subjects were prescribed an exercise regimen of $1.5 \mathrm{MJ} / \mathrm{d}$, which would amount to $10.5 \mathrm{MJ} / \mathrm{week}$ or $42 \mathrm{MJ}$ over the course of the 4-week intervention. This cumulative energy deficit due to the prescribed level of exercise would have produced a weight loss of $1.1 \mathrm{~kg}$ (assuming an energy cost of weight loss of $26 \cdot 2 \mathrm{MJ} / \mathrm{kg}$ ), if no change occurred in either EI or EE during parts of the day when subjects were not exercising. In essence, the women mis-reported their food intake and also failed to achieve their exercise goals.

Under conditions in which subjects were required to exercise under mandatory supervised conditions, the subjects completed an average of nineteen of twenty exercise sessions. However, they only achieved approximately $70 \%$ of the prescribed exercise levels. The difficulty the women experienced in achieving even these mild levels of exertion clearly limited the success of the exercise intervention. It cannot be deduced from these data whether the subjects felt unable or disinclined to exercise to their required level. Anecdotally the overweight women complained that they found this level of exertion arduous. It is highly likely that if subjects were simply requested to attend a gym without supervision or quantification of their exercise sessions, they would have been even less successful at raising their daily EE. Indeed, of the original twenty-five women who began the study, almost 
one-third failed to successfully complete the exercise intervention. These data suggest a group of subjects who are already overweight, and probably tracking towards clinical obesity in later years, are unlikely to substantially raise total daily EE or benefit body weight when prescribed an exercise regimen in accordance with current World Health Organization (1990) and government recommendations. In general, this vulnerable group appeared to be both insufficiently fit and/or motivated to achieve their exercise goals. These data support recent contentions that exercise prescriptions per se are insufficient to control weight in subjects who are already overweight (Garrow \& Summerbell, 1995; Miller et al. 1997). The effects of exercise are clearly beneficial if an exercise programme is adhered to. However, unless the behavioural feasibility of these prescriptions can be improved, they will be of academic interest with little practical effect.

In conclusion, short (1-2d)-term and medium (7-16d)term studies demonstrate that individuals can tolerate substantial activity-induced negative energy balances of $\leq 4 \mathrm{MJ} / \mathrm{d}$. However, subsequently food intake begins to increase in order to provide compensation for about $30 \%$ of the energy expended in activity. This compensation $(\leq 16 \mathrm{~d})$ is partial and incomplete. Moreover, subjects separate into compensators and non-compensators. The exact nature of these differences in compensation and whether it reflects the maintenance of a habitual eating pattern remains to be determined. Some subjects (men and women) performing activity with a cost of $\leq 4 \mathrm{MJ} / \mathrm{d}$ for $14 \mathrm{~d}$ show no change in daily energy intake. The next stage is to further characterise the compensators and non-compensators, and to identify the mechanisms (physiological or behavioural) that are responsible for the rate of compensation and its limits.

\section{References}

Black AE, Coward WA, Cole TJ \& Prentice AM (1996) Human energy expenditure in affluent societies: an analysis of 574 doubly-labelled water measurements. European Journal of Clinical Nutrition 50, 72-92.

Blundell JE \& King NA (1996) Overconsumption as a cause of weight gain: behavioural-physiological interactions in the control of food intake (appetite). In The Origins and Consequences of Obesity, pp. 138-158 [DJ Chadwick and G Cardew, editors]. Chichester, West Sussex: Wiley.

Blundell JE \& King NA (1998) Effects of exercise on appetite control: loose coupling between energy expenditure and energy intake. International Journal of Obesity 22, 1-8.

Blundell JE \& Stubbs RJ (1998) Diet composition and the control of food intake in humans. In Handbook of Obesity, pp. 243-272[GA Bray, C Bouchard and WPT James, editors]. New York: Marcel Dekker Inc.

Brouns F, Saris WH, Stroecken J, Beckers E, Thijssen R, Rehrer NJ \& ten Hoor F (1989) Eating, drinking, and cycling. A controlled Tour de France simulation study, Part I. International Journal of Sports Medicine 10, S32-S40.

Delargy HD, Burley VJ, Sullivan KR, Fletcher RJ \& Blundell JE (1995) Effects of different soluble:insoluble fibre ratios at breakfast on 24-h pattern of dietary intake and satiety. European Journal of Clinical Nutrition 49, 754-766.

Durrant M \& Royston J (1982) Effect of exercise on energy intake and eating patterns in lean and obese humans. Physiology and Behaviour 29, 449-454.
Edholm OG (1977) Energy balance in man: studies carried out by the Division of Human Physiology, National Institute for Medical Research. Journal of Human Nutrition 31, 413-431.

Edholm OG, Adam JM, Healy MJR, Wolff HS, Goldsmith R \& Best TW (1970) Food intake and energy expenditure of army recruits. British Journal of Nutrition 24, 1091-1107.

Edholm OG, Fletcher JG, Widdowson EM \& McCance RA (1955) The energy expenditure and food intake of individual men. British Journal of Nutrition 9, 286-300.

Elia M, Fuller NJ \& Murgatroyd PR (1992) Measurement of bicarbonate turnover in humans: applicability to estimation of energy expenditure. American Journal of Physiology 263, E676-E678.

Forbes GB, Kreipe RE \& Lipinski B (1982) Body composition and the energy cost of weight gain. Human Nutrition Clinical Nutrition 36C, 485-487.

Garrow JS \& Summerbell CD (1995) Meta-analysis: effect of exercise, with or without dieting, on the body composition of overweight subjects. European Journal of Clinical Nutrition 49, 1-10.

Gibney ER (2001) The physical, psychological and metabolic effects of nutritional depletion and subsequent repletion. $\mathrm{PhD}$ Thesis, University of Cambridge.

Green SM, Burley SM \& Blundell JE (1994) Effect of fat- and sucrose-containing foods on the size of eating episodes and energy intake in lean males: potential for causing overconsumption. European Journal of Clinical Nutrition 48, $547-555$

Health Education Authority and Sports Council (1992) The Allied Dunbar National Fitness Survey. London: Allied Dunbar.

Horton TJ, Drougas H, Brachey A, Reed GW, Peters JC \& Hill JO (1995) Fat and carbohydrate overfeeding in humans: different effects on energy storage. American Journal of Clinical Nutrition 62, 19-29.

Hubert P, King NA \& Blundell JE (1998) Uncoupling the effects of energy expenditure and energy intake: appetite response to shortterm energy deficit induced by meal omission and physical activity. Appetite 31, 9-19.

Hughes DA, Johnstone AM, Horgan GW, King N, Blundell JE \& Stubbs RJ (2003) The effects of a four-week exercise intervention on food intake and energy balance in normal weight and overweight women. European Journal of Clinical Nutrition (In the Press).

Imbeault P, Saint-Pierre S, Almeras N \& Tremblay A (1997) Acute effects of exercise on energy intake and feeding behaviour. British Journal of Nutrition 77, 511-521.

Johnstone AM (2001) Weight loss in human obesity. PhD Thesis, Aberdeen University.

Kant AK, Graubard BI, Schatzkin A \& Ballard-Barbash R (1995) Proportion of energy intake from fat and subsequent weight change in the NHANES Epidemiological Follow-up Study. American Journal of Clinical Nutrition 61, 11-17.

Kelly SM, Shorthouse M, Cotterell JC, Riordan AM, Lee AJ, Thurnham DI, Hanka R \& Hunter JO (1998) A 3-month, doubleblind, controlled trial of feeding with sucrose polyester in human volunteers. British Journal of Nutrition 80, 41-49.

Keys A (1950) The Biology of Human Starvation, Minneapolis, MN: University of Minnesota Press.

King NA (1998) The relationship between physical activity and food intake. Proceedings of the Nutrition Society 57, 1-9.

King NA, Appleton K, Rogers PJ \& Blundell JE (1999) Effects of sweetness and energy in drinks on food intake following exercise. Physiology and Behavior 6, 375-379.

King NA \& Blundell J (1995) High-fat foods overcome the energy expenditure due to exercise after cycling and running. European Journal of Clinical Nutrition 49, 114-123.

King NA, Burley VJ \& Blundell JE (1994) Exercise-induced suppression of appetite: Effects on food intake and implications 
for energy balance. European Journal of Clinical Nutrition $\mathbf{4 8 ,}$ $715-724$.

King NA, Lluch A, Stubbs RJ \& Blundell JE (1997a) High dose exercise does not increase hunger or energy intake in free living males. European Journal of Clinical Nutrition 51, 478-483.

King NA, Snell L, Smith RD \& Blundell JE (1996) Effects of shortterm exercise on appetite response in unrestrained females. European Journal of Clinical Nutrition 50, 663-667.

King NA, Tremblay A \& Blundell JE (1997b) Effects of exercise on appetite control: implications for energy balance. Medicine and Science in Sports and Exercise 29, 1076-1089.

Kissileff HR, Pi-Sunyer XF, Segal K, Meltzer S \& Foelsch PA (1990) Acute effects of exercise on food intake in obese and non-obese women. American Journal of Clinical Nutrition 52, 240-245.

Lane HW, Gretebeck RJ, Schoeller DA, Davis-Street J, Socki RA \& Gibson EK (1997) Comparison of ground-based and space flight energy expenditure and water turnover in middle-aged healthy male US astronauts. American Journal of Clinical Nutrition 65, 4-12.

Lawton CL, Burley VJ, Wales JK \& Blundell JE (1993) Dietary fat and appetite control in obese subjects: weak effects on satiation and satiety. International Journal of Obesity 17, 409-416.

Lim CL \& Lee LK (1994) The effects of 20 weeks basic military training program on body composition, VO2max and aerobic fitness of obese recruits. Journal of Sports Medicine and Physical Fitness 34, 271-278.

Lluch A, King NA \& Blundell JE (1998) Exercise in dietary restrained women: no effect on energy intake but change in hedonic ratings. European Journal of Clinical Nutrition 52, 300-307.

Long SJ, Hart K \& Morgan LM (2002) The ability of habitual exercise to influence appetite and food intake in response to highand low-energy pre-loads in man. British Journal of Nutrition $\mathbf{8 7}$, 517-523.

Maldonado S, Mujika I \& Padilla S (2002) Influence of body mass and height on the energy cost of running in highly trained middle- and long-distance runners. International Journal of Sports Medicine 23, 268-272.

Martinez-Gonzalez MA, Martinez JA, Hu FB, Gibney MJ \& Kearney J (1999) Physical inactivity, sedentary lifestyle and obesity in the European Union. International Journal of Obesity and Related Metabolic Disorders 23, 1192-1201.

Miller WC, Koceja DM \& Hamilton EJ (1997) A meta-analysis of the past 25 years of weight loss research using diet, exercise or diet plus exercise intervention. International Journal of Obesity and Related Metabolic Disorders 21, 941-947.

Murgatroyd PR, Goldberg GR, Leahy FE, Gilsenan MB \& Prentice AM (1999) Effects of inactivity and diet composition on human energy balance. International Journal of Obesity and Related Metabolic Disorders 23, 1269-1275.

Reger WE, Allison TA \& Kurucz RL (1984) Exercise, postexercise metabolic rate and appetite. Sport Health and Nutrition $2,115-123$

Ritz P, Maillet A, Blanc S \& Stubbs RJ (1999) Observations in energy and macronutrient intake during prolonged bed-rest in a head-down tilt position. Clinical Nutrition 18, 203-207.

Saris WH (2002) Does response of physical activity in the treatment of obesity - How much is enough to prevent unhealthy weight gain - Outcome of the first Mike Stock Conference. International Journal of Obesity 26, Suppl. 1, S108.

Schoeller DA (1998) Balancing energy expenditure and body weight. American Journal of Clinical Nutrition 68, Suppl., 956S-961S.

Schoeller DA, Shay K \& Kushner RF (1997) How much physical activity is needed to minimize weight gain in previously obese women? American Journal of Clinical Nutrition 66, 551-556.

Seidell JC (2002) Prevalence and time trends of obesity in Europe. Journal of Endocrinological Investigations 25, 816-822.

Shephard TY, Weil KM, Sharp TA, Grunwald GK, Bell ML, Hill JO \& Eckel RH (2001) Occasional physical inactivity combined with a high-fat diet may be important in the development and maintenance of obesity in human subjects. American Journal of Clinical Nutrition 73, 703-708.

Shephard RJ \& Lavallee H (1978) Physical Fitness Assessment Principles, Practice and Applications. Springfield, IL: CC Thomas.

Stricker EM (2000) Specific appetites and homeostatic systems. In Neural and Metabolic Control of Macronutrient Intake, pp. 856-872 [H-R Berthoud and R Seeley, editors]. Boca Raton, FL: CRC Press.

Stricker EM \& Verbalis JG (1999) Fluid intake and homeostasis. In Fundamental Neuroscience, pp. 1091-1099 [MJ Zigmond, FE Bloom, SC Landis, JL Roberts and LR Squire, editors]. San Diego, CA: Academic Press.

Stubbs RJ \& Elia M (2001) Macronutrients and appetite control with implications for the nutritional management of the malnourished. Clinical Nutrition 20, Suppl. 1, 129-139.

Stubbs RJ, Hughes DA, Johnstone AM, Horgan GW, King N, Elia M \& Blundell JE (2003a) Interactions between energy intake and expenditure in the development and treatment of obesity. Progress in Obesity Research (In the Press).

Stubbs RJ, Hughes DA, Johnstone AM, Horgan GW, King N, Elia M \& Blundell JE (2003b) The effect of a decrease in physical activity on appetite, energy and nutrient balance in lean men feeding ad libitum. American Journal of Clinical Nutrition (In the Press).

Stubbs RJ, Hughes DA, Ritz P, Johnstone AM, Horgan GW, King $\mathrm{N} \&$ Blundell JE (2003c) The effect of an incremental increase in exercise on appetite, feeding behaviour and energy balance in lean men and women feeding ad libitum. Obesity Research (In the Press).

Stubbs RJ, Johnstone M, Mazlan N, Mbaiwa SE \& Reid CA (2000) Effect of altering the sensory variety of foods of the same macronutrient content on food intake and body weight in lean and overweight men. European Journal of Clinical Nutrition 55, $1-10$.

Stubbs RJ, Johnstone AM, O'Reilly LM \& Poppitt SD (1998) Methodological issues relating to the measurement of food, energy and nutrient intake in human laboratory-based studies. Proceedings of the Nutrition Society 57, 357-372.

Stubbs RJ, Johnstone A, Rist M, Kracht A \& Reid C (2001) How covert are covertly manipulated diets? International Journal of Obesity and Related Metabolic Disorders 25, 567-573.

Stubbs RJ, Sepp A, Hughes DA, Johnstone AM, Horgan GW, King N \& Blundell J (2002a) The effect of graded levels of exercise on energy intake and balance in free-living men, consuming their normal diet. European Journal of Clinical Nutrition 56, 129-140.

Stubbs RJ Sepp A, Hughes DA, Johnstone AM, King N, Horgan G \& Blundell JE (2002b) The effect of graded levels of exercise on energy intake and balance in free-living women. International Journal of Obesity and Related Metabolic Disorders 26, 866-869.

Thompson DA, Wolfe LA \& Eikelboom R (1988) Acute effects of exercise intensity on appetite in young men. Medicine and Science in Sports and Exercise 20, 222-227.

Tremblay A, Despres JP \& Bouchard C (1985) The effects of exercise-training on energy balance and adipose tissue morphology and metabolism. Sports Medicine 2, 223-233.

Van Baak MA (1999) Physical activity and energy balance. Public Health Nutrition 2, 335-339. 
Van Etten LM, Westerterp KR, Verstappen FT, Boon BJ \& Saris WH (1997) Effect of an 18-wk weight-training program on energy expenditure and physical activity. Journal of Applied Physiology 82, 298-304.

Verboeket-van de Venne W P, Westerterp KR, HermansLimpens TJ, de Graaf C, van het Hof KH \& Weststrate JA (1996) Long-term effects of consumption of full-fat or reduced-fat products in healthy non-obese volunteers: assessment of energy expenditure and substrate oxidation. Metabolism 45, 1004-1010.

Westerterp KR (1998) Alterations in energy balance with exercise. American Journal of Clinical Nutrition 68, 970S-974S.

Westerterp-Plantenga MS, Verwegen CRT, Ijedema MJW, Wijckmans NEG \& Saris WHM (1997) Acute effects of exercise or sauna on appetite in obese and non-obese men. Physiology and Behaviour 62, 1345-1354.

Woo R, Garrow JS \& Pi-Sunyer FX (1982a) Effect of exercise on spontaneous calorie intake in obesity. American Journal of Clinical Nutrition 36, 470-477.

Woo R, Garrow JS \& Pi-Sunyer FX (1982b) Voluntary food intake during prolonged exercise in obese women. American Journal of Clinical Nutrition 36, 478-484.

Woo R \& Pi-Sunyer FX (1985) Effect of increased physical activity on voluntary intake in lean women. Metabolism 34, 836-41.

World Health Organization (1990) Diet, Nutrition and the Prevention of Chronic Diseases. Technical Report Series no 797. Geneva: WHO. 